This is an Author's Accepted Manuscript of an article published as: COSTA, J.L. (2010) Proof of Dain inequality with charge. Journal of Physics A: Mathematical and Theoretical. 43(28):285202 available online at:

http://dx.doi.org/10.1088/1751-8113/43/28/285202 


\title{
Proof of a Dain Inequality with charge
}

\author{
João Lopes Costa \\ Lisbon University Institute - ISCTE \\ Mathematical Institute and Magdalen College, Oxford
}

May 20, 2010

\begin{abstract}
We prove an upper bound for angular-momentum and charge in terms of the mass for electro-vacuum asymptotically flat axisymmetric initial data sets with simply connected orbit space. This completes the work started in [7] where this charged Dain inequality was first presented but where the proof of the main result, based on the methods of [5], was only sketched. Here we present a complete proof while simplifying the methods suggested by [7].
\end{abstract}

\section{Introduction}

Gravitational collapse involving suitable $e^{1}$ matter is expected $[11,17,18]$ to generically result in the formation of an event horizon whose exterior solution approaches a Kerr-Newman metric asymptotically with time, here we are assuming that the exterior region becomes electro-vacuum. Then, the characteristic inequality

$$
m_{\infty} \geq \sqrt{\frac{\left|\overrightarrow{J_{\infty}}\right|^{2}}{m_{\infty}^{2}}+Q_{E, \infty}^{2}+Q_{B, \infty}^{2}}
$$

\footnotetext{
${ }^{1}$ What constitutes suitable matter is part of the challenge posed by the cosmic censorship conjectures, but some restrictions are clear, most notably, one should restrict to matter models which do not develop singularities in the absence of gravity. For a detailed discussion see [18].
} 
relating the mass, angular momentum and the Maxwell charges of such blackholes should also be valid asymptotically with time. Now, mass is nonincreasing while the Maxwell charges are conserved quantities. If one further assumes axisymmetry we are able to define the angular momentum using a Komar integral which is also conserved, see, e.g., [7]. So, letting $m, \vec{J}, Q_{E}$ and $Q_{B}$ denote the Poncaré and Maxwell charges of axisymmetric initial data for such a collapse we see that

$$
m \geq m_{\infty} \geq \sqrt{\frac{\left|\vec{J}_{\infty}\right|^{2}}{m_{\infty}^{2}}+Q_{E, \infty}^{2}+Q_{B, \infty}^{2}} \geq \sqrt{\frac{|\vec{J}|^{2}}{m^{2}}+Q_{E}^{2}+Q_{B}^{2}} .
$$

Besides their own intrinsic interest, results establishing such inequalities provide evidences in favor of this "current standard picture of gravitational collapse" [11], which is based upon weak cosmic censorship and a version of black hole uniqueness considerably stronger than the ones available $[6,8,10]$.

Dain $[12,13]$, besides providing the previous Penrose-like heuristic argument, proved an upper bound for angular-momentum in terms of the mass for a class of maximal, vacuum, axisymmetric initial data sets. The analysis of [12] has been extended in [5] to include vacuum axisymmetric initial data, with simply connected orbit space, and manifolds which are asymptotically flat in the standard sense, allowing moreover several asymptotic ends. Recently a generalized Dain's inequality including electric and magnetic charges was obtained in [7]; there the proof of the main result, based on the methods of [5], was only sketched. The aim of this work is to provide a complete proof of this charged Dain inequality while simplifying the methods of [5]. A few comments concerning the refereed simplifications are in order: Here, we have been able to follow the strategy developed in [5] by relaying solely on the estimates arising from asymptotic flatness and the inversion procedure (to be described briefly, see (2.20)). By doing so, we have eliminated the necessity of introducing an extra family of auxiliary maps (denoted by $\check{U}_{\mu}$ in [5]) and we were also able to proceed without relaying on the weighted Poincaré inequality provided by Proposition 2.4 of [5]. It should be noted that while the introduction of charge leads to a more complicated mass functional (2.13), dealing with an arbitrary finite number of asymptotically flat ends, as dealt with in [5], leads to other difficulties, most notably the fact that an explicit form for the comparison map, here the map defined by extreme Kerr-Newman, is longer available in general. Nonetheless, the simplifications presented here may easily be implemented in [5] without weakening the 
results established there.

We will prove the following (we refer to Section 2 for the necessary intermediary definitions and to $[9,10]$ for a more detailed exposition):

Theorem 1.1 Let $(M, g, K, E, B)$ be a three dimensional, electro-vacuum, smooth data set invariant under an action of $\mathrm{U}(1)$, where $M$ is the union of a compact set and of two asymptotically flat regions $M_{1}$ and $M_{2}$, in the sense of (2.2) and (2.3), with $k \geq 6$. Let $m, \vec{J}, Q_{E}$ and $Q_{B}$ denote respectively the $A D M$ mass, the ADM angular momentum and the total electric and magnetic charges of $M_{1}$. If $M / U(1)$ is simply connected, then

$$
m \geq \sqrt{\frac{|\vec{J}|^{2}}{m^{2}}+Q_{E}^{2}+Q_{B}^{2}} .
$$

REMARK 1.2 We expect the equality to be attained only for the magnetically and electrically charged extreme Kerr-Newman space-times, which do not satisfy the hypotheses of Theorem 1.1. Indeed, any spacelike manifold in an extreme Kerr-Newman space-time is either incomplete, or contains a boundary, or a singularity, or an asymptotically cylindrical end.

\section{A lower bound for the ADM mass of electro- vacuum and axisymmetric initial data}

An electro-vacuum initial data set is a quintuplet $(M, g, K, E, B)$, with $(M, g)$ a Riemannian manifold, $K$ a symmetric two-tensor on $M$, and $E, B \in T M$ divergence free, satisfying the Einstein-Maxwell scalar constraint equation, which, for maximal initial data $\left(\operatorname{tr}_{g} K=0\right)$, reads

$$
{ }^{(3)} R=16 \pi \mu+|K|_{g}^{2}+2\left(|E|_{g}^{2}+|B|_{g}^{2}\right) .
$$

In the previous equation, ${ }^{(3)} R$ is the scalar curvature of $g$, the function $\mu \geq 0$ represents the non-electromagnetic energy density and $|\cdot|_{g}$ denotes the norm with respect to the metric $g$.

Recall also that an asymptotically flat end is a region $M_{\text {ext }} \subset M$ diffeomorphic to $\mathbb{R}^{3} \backslash B(R)$, where $B(R)$ is a coordinate ball of radius $\mathrm{R}$, such that 
in coordinates on $M_{\text {ext }}$ obtained from $\mathbb{R}^{3} \backslash B(R)$ we have, for some $k \geq 1{ }^{2}$

$$
g_{i j}=\delta_{i j}+o_{k}\left(r^{-1 / 2}\right), \partial_{k} g_{i j} \in L^{2}\left(M_{\mathrm{ext}}\right), \quad K_{i j}=O_{k-1}\left(r^{-\beta}\right), \beta>\frac{5}{2} .
$$

It will be necessary to request that, in the previous asymptotically flat coordinates, ${ }^{3}$

$$
E^{i}=O_{k-1}\left(r^{-\gamma-1}\right), \quad B^{i}=O_{k-1}\left(r^{-\gamma-1}\right), \gamma>3 / 4
$$

We will be dealing with axisymmetric initial data, i.e., data which is invariant under an action of $\mathrm{U}(1)$. By further assuming that $M / U(1)$ is simply connected we are able to rely on $[3]^{4}$ for the existence of a coordinate system, with controlled asymptotic behavior, in which the metric takes the form

$$
g=e^{-2 U+2 \alpha}\left(d \rho^{2}+d z^{2}\right)+\rho^{2} e^{-2 U}\left(d \varphi+\rho W_{\rho} d \rho+W_{z} d z\right)^{2},
$$

where

$$
\eta:=\partial_{\varphi},
$$

is the axial Killing vector; such coordinates are global in $M_{1}$, with $M_{2}$ being represented by the "puncture" $\{\rho=z=0\}$.

To obtain our inequality we start by bounding the ADM mass from below by a mass functional depending on $U$, the unknown in (2.4) determining the norm of the axial Killing vector, and on global (electromagnetic) potentials $\chi, \psi$ and $v$ whose definition we will now recall: The electric and magnetic fields $E$ and $B$ are the orthogonal projections to $T M$ of their space-time analogues

$$
E^{\mu}=F^{\mu}{ }_{\nu} n^{\nu}, \quad B^{\mu}=* F^{\mu}{ }_{\nu} n^{\nu},
$$

where $F$ is the Maxwell two-form, and where $n$ is a unit normal to $M$, when embedded in an electro-vacuum space-time. ${ }^{5}$ Then, relaying on Maxwell's

\footnotetext{
${ }^{2}$ We write $f=o_{k}\left(r^{-\alpha}\right)$ if the $\operatorname{limits} \lim _{r \rightarrow \infty} r^{\alpha+\ell} \partial_{k_{1}} \ldots \partial_{k_{\ell}} f$ vanish for all $0 \leq \ell \leq k$, and $f=O_{k}\left(r^{-\alpha}\right)$ if there exists a constant $C$ such that $\left|r^{\alpha+\ell} \partial_{k_{1}} \ldots \partial_{k_{\ell}} f\right| \leq \bar{C}$ for all $0 \leq \ell \leq k$.

${ }^{3}$ The need to impose this unusual ranges for $\beta$ and $\gamma$ will became apparent in the proof of the main result, see, e.g., (2.24). Also, an identical decay rate for the second fundamental form was already needed in vacuum [5].

${ }^{4}$ To invoke [3] we also need to assume asymptotic flatness with $k \geq 6$.

${ }^{5}$ The existence of an electro-vacuum and axisymmetric evolution of the data follows from its smoothness by [2] and [4]. This will, in particular, allow us to use the (space-time) computations in [19].
} 
equations, axisymmetry and simple-connectedness of $M / U(1)$, we are able to find $\chi, \psi, v: M \rightarrow \mathbb{R}$ satisfying

$$
\partial_{\alpha} \chi=F_{\mu \alpha} \eta^{\mu}, \quad \partial_{\alpha} \psi=* F_{\mu \alpha} \eta^{\mu}
$$

and

$$
d v=\frac{1}{2} \lambda-\chi d \psi+\psi d \chi
$$

where

$$
\lambda:=2 \epsilon_{i j k} K^{j}{ }_{\ell} \eta^{k} \eta^{\ell} d x^{i} .
$$

The desired lower bound on mass was established in [7], based on the results of $[3]$ (compare $[1,12,15])$, and reads

$m \geq \frac{1}{8 \pi} \int\left[(D U)^{2}+\frac{e^{4 U}}{\rho^{4}}(D v+\chi D \psi-\psi D \chi)^{2}+\frac{e^{2 U}}{\rho^{2}}\left((D \chi)^{2}+(D \psi)^{2}\right)\right] d^{3} x$

where, from now on, we use the symbol $D f$ to denote the gradient of a function $f$ with respect to the flat metric $\delta=d \rho^{2}+d z^{2}+\rho^{2} d \varphi^{2}$, and we will use both $(v)^{2}$ and $|v|^{2}$ in alternative to $|v|_{\delta}^{2}$, the squared norm of $v$ with respect to $\delta$.

REMARK 2.1 As discussed in [9], see also [11], for stationary data with vanishing non-electromagnetic energy density $(\mu=0)$ equality is attained in $(2.9)$.

We have the following, from which Theorem 1.1 immediately follows:

TheOrem 2.2 Let $(M, g, K, v, \chi, \psi)$ be a three dimensional smooth data set invariant under an action of $\mathrm{U}(1)$, where $M$ is the union of a compact set and of two asymptotically flat regions $M_{1}$ and $M_{2}$, in the sense of (2.2), with $k \geq 6$, and where $v, \psi$ and $\chi$ are global potentials as in (2.6) and (2.7), satisfying (2.3). Let $m$ and $\vec{J}$ denote the ADM mass and ADM angular momentum of $M_{1}$, and let $Q_{E}$ and $Q_{B}$ be the global electric and magnetic charges of $M_{1}$. If (2.9) holds and $M / \mathrm{U}(1)$ is simply connected, then

$$
m \geq \sqrt{\frac{|\vec{J}|^{2}}{m^{2}}+Q_{E}^{2}+Q_{B}^{2}} .
$$


REMARK 2.3 We stress the fact that, in the previous result, no constraints are assumed. Nonetheless the parallelism with Einstein-Maxwell is clear and the use of the electromagnetic terminology seems appropriate.

For future reference we take the chance to provide formulas for the Maxwell 2 -form and the global charges in terms of the axial potentials: in an orthonormal basis $\left\{n, e_{i}\right\}$, with $e_{i} \in T M, e_{3}$ proportional to $\eta$, and $n$ normal to $M$, when embedded in a space-time not necessarily satisfying Einstein equations, the Maxwell 2-form is given by

$$
F_{\mu \nu}=\frac{e^{U}}{\rho}\left(\begin{array}{cccc}
0 & \partial_{2} \psi & -\partial_{1} \psi & -\partial_{1} \chi \\
-\partial_{2} \psi & 0 & 0 & -\partial_{2} \chi \\
\partial_{1} \psi & 0 & 0 & 0 \\
\partial_{1} \chi & \partial_{2} \chi & 0 & 0
\end{array}\right)
$$

Since $\psi, \chi$ and $v$ are constant on each connected component $\mathscr{A}_{j}$ of the "axis", we set

$$
v_{j}:=\left.v\right|_{\mathscr{A}_{j}}, \quad \psi_{j}:=\left.\psi\right|_{\mathscr{A}_{j}}, \quad \chi_{j}:=\left.\chi\right|_{\mathscr{A}_{j}}, \quad j=1,2,
$$

where $\mathscr{A}_{1}=\{\rho=0\} \cap\{z<0\}$ and $\mathscr{A}_{2}=\{\rho=0\} \cap\{z>0\}$. Then, using equations (2.6) we see that (compare [19])

$$
\begin{aligned}
Q_{E} & :=-\frac{1}{4 \pi} \int_{S_{\infty}} * F=-\frac{-2 \pi}{4 \pi} \int_{S_{\infty} / U(1)} i_{\eta} * F \\
& =\frac{1}{2} \int_{S_{\infty} / U(1)} d \psi=\frac{\psi_{2}-\psi_{1}}{2}
\end{aligned}
$$

with a similar computation yielding

$$
Q_{B}:=\frac{1}{4 \pi} \int_{S_{\infty}} F=\frac{\chi_{1}-\chi_{2}}{2}
$$

Proof: If the mass is infinite there is nothing to prove, otherwise by $(2.9)$ we need to find a lower bound on

$$
I:=\int_{\mathbb{R}^{3}}\left[(D U)^{2}+\frac{e^{4 U}}{\rho^{4}}(D v+\chi D \psi-\psi D \chi)^{2}+\frac{e^{2 U}}{\rho^{2}}\left((D \chi)^{2}+(D \psi)^{2}\right)\right] d^{3} x .
$$

Let $(\tilde{U}, \tilde{v}, \tilde{\chi}, \tilde{\psi})$ be the map associated with the extreme Kerr-Newman with angular momentum along the $z$-axis equal to $\left(v_{2}-v_{1}\right) / 8$ and electric charge $\left(\psi_{2}-\psi_{1}\right) / 2$. We wish to show that the action $I:=I(U, v, \chi, \psi)$ 
is larger than or equal to that of $(\tilde{U}, \tilde{v}, \tilde{\chi}, \tilde{\psi})$, which shall be denoted by $\tilde{I}$. As we shall see $I$ differs form an harmonic map action $H(2.33)$ by a boundary term; the idea is then to use a result of [16], that the action $H$ is minimized by the solution of the Dirichlet problem which is expected to be $(\tilde{U}, \tilde{v}, \tilde{\chi}, \tilde{\psi})$; however, that result does not apply directly because of the singularity of the equations at the axis $\rho=0$; moreover, we are working in an unbounded domain. We will overcome such problems by constructing a controlled sequence of integrals over compact domains which avoid the singular set and saturate $\mathbb{R}^{3}$. Such strategy was developed in [5]; here we generalize it to the electro-vacuum setting.

So, let $\sigma>0, r=\sqrt{\rho^{2}+z^{2}}$ and let $f_{\sigma} \in C^{\infty}\left(\mathbb{R}^{3}\right)$ be any family of functions satisfying

- $\partial_{\varphi} f_{\sigma} \equiv 0$;

- $0 \leq f_{\sigma} \leq 1$;

- $f_{\sigma}=0$ on the set $\{r \leq \sigma / 2\} \cup\{r \geq 2 / \sigma\}$;

- $f_{\sigma}=1$ on the set $\{r \geq \sigma\} \cap\{r \leq 1 / \sigma\}$;

- $\left|D f_{\sigma}\right| \leq C / \sigma$ for $\sigma / 2 \leq r \leq \sigma$; and

- $\left|D f_{\sigma}\right| \leq C \sigma$ for $1 / \sigma \leq r \leq 2 / \sigma$.

Let $\theta=U, v, \chi, \psi$ and write

$$
\theta_{\sigma}:=f_{\sigma} \theta+\left(1-f_{\sigma}\right) \tilde{\theta}=f_{\sigma}(\theta-\tilde{\theta})+\tilde{\theta} .
$$

We claim that $I^{\sigma}:=I\left(U_{\sigma}, v_{\sigma}, \chi_{\sigma}, \psi_{\sigma}\right)$ satisfies

LEMma $2.4 \lim _{\sigma \rightarrow 0} I^{\sigma}=I$.

Proof: Indeed, for

$$
\lambda_{\sigma}:=D v_{\sigma}+\chi_{\sigma} D \psi_{\sigma}-\psi_{\sigma} D \chi_{\sigma},
$$


we have

$$
\begin{aligned}
\int_{\mathbb{R}^{3}} \frac{e^{4 U_{\sigma}}}{\rho^{4}}\left|\lambda_{\sigma}\right|^{2}= & \underbrace{\int_{\{0 \leq \sigma / 2\}} \frac{e^{4 \tilde{U}}}{\rho^{4}}|\tilde{\lambda}|^{2}}_{I}+\underbrace{\int_{\{\sigma / 2 \leq r \leq \sigma\}} \frac{e^{4 U_{\sigma}}}{\rho^{4}}\left|\lambda_{\sigma}\right|^{2}}_{I I}+ \\
& +\underbrace{\int_{\{\sigma \leq r \leq 1 / \sigma\}} \frac{e^{4 U}}{\rho^{4}}|\lambda|^{2}}_{I I I}+\underbrace{\int_{\{1 / \sigma \leq r \leq 2 / \sigma\}} \frac{e^{4 U_{\sigma}}}{\rho^{4}}\left|\lambda_{\sigma}\right|^{2}}_{I V} \\
& +\underbrace{\int_{\{2 / \sigma \leq r\}} \frac{e^{4 \tilde{U}}}{\rho^{4}}|\tilde{\lambda}|^{2}}_{V} .
\end{aligned}
$$

Since the maps under consideration have finite energy, the integrals $I$ and $V$ converge to zero, by the dominated convergence theorem. III converges to the integral over $\mathbb{R}^{3}$ of $\frac{e^{4 U}}{\rho^{4}}|\lambda|^{2}$ by, e.g., the monotone convergence theorem.

We will now show that

$$
I I=\int_{\{\sigma / 2 \leq r \leq \sigma\}} \frac{e^{4 U_{\sigma}}}{\rho^{4}}\left(D v_{\sigma}+\chi_{\sigma} D \psi_{\sigma}-\psi_{\sigma} D \chi_{\sigma}\right)^{2} \rightarrow_{\sigma \rightarrow 0} 0 .
$$

The key identity is ${ }^{6}$

$$
\begin{aligned}
\lambda_{\sigma}= & f_{\sigma} \lambda+\left(1-f_{\sigma}\right) \tilde{\lambda}+D f_{\sigma}(v-\tilde{v})+D f_{\sigma}(\tilde{\chi} \psi-\tilde{\psi} \chi) \\
& +f_{\sigma}\left(1-f_{\sigma}\right)\{(\psi-\tilde{\psi}) D(\chi-\tilde{\chi})-(\chi-\tilde{\chi}) D(\psi-\tilde{\psi})\},
\end{aligned}
$$

which will allow to establish (2.15) by a step-by-step estimation of the integrals obtained by replacing $\lambda_{\sigma}$ by each of its five summands. We will exemplify this by dealing with the most delicate case.

The existence of multiple ends manifests itself in the asymptotic behavior

$$
U=2 \log r+O(1), r \rightarrow 0,
$$

established in [3, Theorem 2.9, p. 2580]. Then, using the decay rates of the extreme Kerr-Newman map, compiled in Table 1 of Appendix A, we get ${ }^{7}$

$$
\begin{aligned}
e^{4 U_{\sigma}} & =e^{4 f_{\sigma} U} e^{4\left(1-f_{\sigma}\right) \tilde{U}} \\
& \lesssim r^{8 f_{\sigma}} r^{4\left(1-f_{\sigma}\right)} \\
& =r^{4\left(f_{\sigma}+1\right)} \leq r^{4}, r \rightarrow 0 .
\end{aligned}
$$

\footnotetext{
${ }^{6}$ The computations leading to this identity are presented in Appendix B.

${ }^{7}$ We will write $f \lesssim g$ if and only if $f=O(g)$.
} 
From (2.8) and asymptotic flatness (compare [3, Theorem 2.7, p. 2580]) we see that

$$
|\lambda|_{\delta}=\rho^{2} O\left(r^{-\beta}\right), r \rightarrow+\infty .
$$

To control the behavior for small $r$ we proceed as follows: Recall that near $r=$ 0 the coordinates $(\rho, z)$ can be obtained from the usual cylindrical coordinates in the other asymptotically flat region, which we denote by $(\hat{\rho}, \hat{z})$, by an inversion $(\hat{\rho}, \hat{z})=\left(\frac{\rho}{r^{2}}, \frac{z}{r^{2}}\right)$ (see the proof of [3, Theorem 2.9]). This leads to estimates for small $r$, equivalently for large $\hat{r}$, such as

$$
|\lambda|_{\delta}=\frac{1}{r^{2}}|\lambda|_{\hat{\delta}} \lesssim \frac{1}{r^{2}} \hat{\rho}^{2} \hat{r}^{-\beta} \lesssim \frac{1}{r^{2}} \frac{\rho^{2}}{r^{4}} r^{\beta}=\rho^{2} r^{\beta-6}, r \rightarrow 0,
$$

where $\hat{\delta}=d \hat{\rho}^{2}+d \hat{z}^{2}+\hat{\rho}^{2} d \varphi^{2}$.

The same procedure yields

$$
|D \chi|_{\delta},|D \psi|_{\delta}=\rho O\left(r^{\gamma-3}\right), r \rightarrow 0
$$

and we see that, for small $r$,

$$
|D v|_{\delta} \leq|\lambda|_{\delta}+|\chi D \psi-\psi D \chi|_{\delta} \lesssim \rho^{2} r^{\beta-6}+\rho r^{2 \gamma-4} .
$$

From this and the known asymptotic behaviour of extreme Kerr-Newman one obtains, when $\beta \geq 2 \gamma+1$,

$$
v-\tilde{v}=O\left(r^{2 \gamma-2}\right)
$$

and we are now able to see that the contribution of the term $D f_{\sigma}(v-\tilde{v})$ in the region $\rho \geq z$, where $r$ is comparable to $\rho$, is estimated by

$$
\int_{\sigma / 2}^{\sigma} \frac{r^{4}}{\rho^{4} r^{2}}\left(r^{2 \gamma-2}\right)^{2} r^{2} d r \lesssim \sigma^{4 \gamma-3} \rightarrow_{\sigma \rightarrow 0} 0 \text { provided that } \gamma>3 / 4
$$

This explains our ranges of $\beta$ and $\gamma$ in $(2.2)$ and $(2.3)^{8}$.

Since $v$ and $\tilde{v}$ have the same axis data, Taylor expanding on $\rho$ along the axis yields

$$
(v-\tilde{v})(\rho, z)=\underbrace{(v-\tilde{v})(0, z)}_{=0}+\partial_{\rho}(v-\tilde{v})(c(\rho), z) \rho,|c(\rho)| \leq|\rho| .
$$

\footnotetext{
${ }^{8}$ For $\beta<2 \gamma+1$ the dominating behaviour in (2.22) is governed by $\lambda$, which leads to $v-\tilde{v}=O\left(r^{\beta-3}\right)$ and the necessity to impose $\beta>5 / 2$, as in vacuum [5, p. 2602].
} 
Also, again for $\beta \geq 2 \gamma+1$,

$$
\partial_{\rho} v=\rho O\left(r^{2 \gamma-4}\right),
$$

with the same estimate holding for the $\rho$-derivative of the difference. Then, in $\{\rho \leq z\}$,

$$
v-\tilde{v}=\rho^{2} O\left(r^{2 \gamma-4}\right), r \rightarrow 0 .
$$

We see that, in this region, the integral under consideration is estimated by

$$
\int_{\{\theta: \rho<z\}} \int_{\sigma / 2}^{\sigma} \frac{r^{4}}{\rho^{4} r^{2}}\left(\rho^{2} r^{2 \gamma-4}\right)^{2} r^{2} \sin \theta d r d \theta \lesssim \sigma^{4 \gamma-3} \rightarrow_{\sigma \rightarrow 0} 0,
$$

and (2.15) follows.

The proof that

$$
I V \rightarrow{ }_{\sigma \rightarrow 0} 0,
$$

follows along the lines of the proof of (2.15) with the necessary estimates for the terms appearing in equation (2.16) following directly from asymptotic flatness. The remaining terms in $I^{\sigma}$ can be controlled in a similar, although considerably more direct and simpler, fashion. For instance, when controlling the $\left|D U_{\sigma}\right|^{2}$ term one of the steps requires to estimate the integral

$$
\begin{aligned}
\int_{\{\sigma / 2 \leq r \leq \sigma\}}\left|D U_{\sigma}\right|^{2} & =\int_{\{\sigma / 2 \leq r \leq \sigma\}}\left|(U-\tilde{U}) D f_{\sigma}+f_{\sigma} D U+\left(1-f_{\sigma}\right) D \tilde{U}\right|^{2} \\
& \lesssim \int_{\{\sigma / 2 \leq r \leq \sigma\}}\left((U-\tilde{U})^{2} r^{-2}+|D U|^{2}+|D \tilde{U}|^{2}\right),
\end{aligned}
$$

where in fact the second and third term go to zero by the Lebesgue dominated convergence theorem while the vanishing of the first follows by direct estimation using (2.17) and the decay rates presented in Table 1.

We now show that:

LEMma $2.5 I^{\sigma} \geq \tilde{I}$ for all $\sigma$ small enough.

Proof: This time consider, for $0<\epsilon<1$,

$$
\hat{f}_{\epsilon}= \begin{cases}0, & \rho \leq \epsilon \\ \frac{\log \frac{\rho}{\epsilon}}{\log \frac{\sqrt{\epsilon}}{\epsilon}}, & \epsilon \leq \rho \leq \sqrt{\epsilon} \\ 1, & \rho \geq \sqrt{\epsilon}\end{cases}
$$


Set, for $\theta=U, v, \chi, \psi$,

$$
\theta_{\sigma, \epsilon}=\hat{f}_{\epsilon} \theta_{\sigma}+\left(1-\hat{f}_{\epsilon}\right) \tilde{\theta},
$$

and let $I^{\sigma, \epsilon}$ denote the action of $\left(U_{\sigma, \epsilon}, v_{\sigma, \epsilon}, \chi_{\sigma, \epsilon}, \psi_{\sigma, \epsilon}\right)$ and

$$
\lambda_{\sigma, \epsilon}=D v_{\sigma, \epsilon}+\chi_{\sigma, \epsilon} D \psi_{\sigma, \epsilon}-\psi_{\sigma, \epsilon} D \chi_{\sigma, \epsilon} .
$$

We claim that

$$
\int_{\{\rho \leq \sqrt{\epsilon}\}}\left[\left(D U_{\sigma, \epsilon}\right)^{2}+\frac{e^{4 U_{\sigma, \epsilon}}}{\rho^{4}}\left(\lambda_{\sigma, \epsilon}\right)^{2}+\frac{e^{2 U_{\sigma, \epsilon}}}{\rho^{2}}\left(\left(D \chi_{\sigma, \epsilon}\right)^{2}+\left(D \psi_{\sigma, \epsilon}\right)^{2}\right)\right] d^{3} x \rightarrow_{\epsilon \rightarrow 0} 0 .
$$

Equivalently,

$$
I^{\sigma, \epsilon} \rightarrow_{\epsilon \rightarrow 0} I^{\sigma} .
$$

In order to see this, note that the integral over the set $\{0 \leq \rho \leq \epsilon\}$, where $\theta_{\sigma, \epsilon}=\tilde{\theta}$, approaches zero as $\epsilon$ tends to zero by the Lebesgue dominated convergence theorem; the same happens away from the set $\{\sigma / 2<r<2 / \sigma\}$. So it remains to consider the integral over

$$
\mathscr{W}_{\sigma, \epsilon}:=\{\epsilon \leq \rho \leq \sqrt{\epsilon}\} \cap\{\sigma / 2<r<2 / \sigma\} .
$$

The computations leading to (2.16) now give

$$
\begin{aligned}
\lambda_{\sigma, \epsilon}= & \hat{f}_{\epsilon} \lambda_{\sigma}+\left(1-\hat{f}_{\epsilon}\right) \tilde{\lambda}+D \hat{f}_{\epsilon}\left(v_{\sigma}-\tilde{v}\right)+D \hat{f}_{\epsilon}\left(\tilde{\chi} \psi_{\sigma}-\tilde{\psi} \chi_{\sigma}\right) \\
& +\hat{f}_{\epsilon}\left(1-\hat{f}_{\epsilon}\right)\left\{\left(\psi_{\sigma}-\tilde{\psi}\right) D\left(\chi_{\sigma}-\tilde{\chi}\right)-\left(\chi_{\sigma}-\tilde{\chi}\right) D\left(\psi_{\sigma}-\tilde{\psi}\right)\right\} .
\end{aligned}
$$

Since $I^{\sigma} \rightarrow I$, we see that $I^{\sigma}$ must be finite, at least for all small enough $\sigma$. Fix such a $\sigma>0$. As before the first two terms in the right-hand side of (2.29) constitute no problem. To control the others note that, for all $\epsilon$ such that $\sqrt{\epsilon}<\sigma / 2$, we have, in the $(\rho, z, \varphi)$ coordinates,

$$
\mathscr{W}_{\sigma, \epsilon} \subseteq[\epsilon, \sqrt{\epsilon}] \times\left(\left[z_{0}(\sigma), z_{1}(\sigma)\right] \cup\left[z_{2}(\sigma), z_{3}(\sigma)\right]\right) \times[0,2 \pi],
$$

for a good choice of $z_{i}$ 's satisfying $z_{i}(\sigma) \neq 0$; e.g., the $z$-coordinate value, in increasing order, of the points in the intersection of $\rho=\epsilon$ with both $r=\sigma / 2$ and $r=2 / \sigma$. We then see that

$$
\begin{aligned}
\int_{\mathscr{W}_{\sigma, \epsilon}} \frac{e^{4 U_{\sigma, \epsilon}}}{\rho^{4}}\left(D \hat{f}_{\epsilon}\left(v_{\sigma}-\tilde{v}\right)\right)^{2} d^{3} x & \leq 2 \pi \sum_{i=0,1} \int_{z_{2 i}(\sigma)}^{z_{2 i+1}(\sigma)} \int_{\epsilon}^{\sqrt{\epsilon}} \frac{e^{4 U_{\sigma, \epsilon}}}{\rho^{4}}\left(D \hat{f}_{\epsilon}\right)^{2}\left(v_{\sigma}-\tilde{v}\right)^{2} \rho d \rho d z \\
& \leq 2 \pi \sum_{i=0,1} \int_{z_{2 i}(\sigma)}^{z_{2 i+1}(\sigma)} \int_{\epsilon}^{\sqrt{\epsilon}} \frac{C(\sigma)}{\rho^{3}} \frac{1}{\rho^{2}(\log \epsilon)^{2}}\left(v_{\sigma}-\tilde{v}\right)^{2} d \rho d z
\end{aligned}
$$


Since $z_{i}(\sigma) \neq 0$ we see that $v_{\sigma}$ and $\tilde{v}$ are smooth on the set $\{\rho \leq \sqrt{\epsilon}, z \in$ $\left.\cup_{i}\left[z_{2 i}, z_{2 i+1}\right]\right\}$. Then, Taylor expanding on $\rho$ along the axis, while noting that $v_{\sigma}$ and $\tilde{v}$ have the same axis data and that $f_{\sigma}$ is, by construction, axisymmetric, yields (compare (2.25))

$$
v_{\sigma}-\tilde{v}=O\left(\rho^{2}\right), \rho \rightarrow 0, \text { in }\left\{\rho \leq \sqrt{\epsilon}, z \in \cup_{i}\left[z_{2 i}, z_{2 i+1}\right]\right\},
$$

hence

$$
\begin{aligned}
2 \pi \sum_{i=0,1} \int_{z_{2 i}(\sigma)}^{z_{2 i+1}(\sigma)} \int_{\epsilon}^{\sqrt{\epsilon}} \frac{C(\sigma)}{\rho^{3}} \frac{1}{\rho^{2}(\log \epsilon)^{2}}(v-\tilde{v})^{2} d \rho d z & \lesssim \frac{C(\sigma)}{(\log \epsilon)^{2}} \int_{\epsilon}^{\sqrt{\epsilon}} \frac{1}{\rho^{5}} \rho^{4} d \rho \\
& \lesssim \frac{C(\sigma)}{(\log \epsilon)^{2}} \log \epsilon \rightarrow_{\epsilon \rightarrow 0} 0 .
\end{aligned}
$$

The remaining terms are controlled in an analogous way, with the $\left(D U_{\sigma, \epsilon}\right)^{2}$ term behaving exactly as in vacuum [5]. This ends the proof of (2.27).

Using the rescaling $U=u+\ln \rho$ we have

$$
I_{\Omega}(U, v, \chi, \psi)=H_{\Omega}(u, v, \chi, \psi)+B_{\Omega}(U),
$$

where

$$
H_{\Omega}=\int_{\Omega}\left[(D u)^{2}+e^{4 u}(D v+\chi D \psi-\psi D \chi)^{2}+e^{2 u}\left((D \chi)^{2}+(D \psi)^{2}\right)\right] d^{3} x,
$$

is the energy, over $\Omega \subset \subset \mathbb{R}^{3} \backslash \mathscr{A}$, of the harmonic map

$$
\Phi=(u, v, \chi, \psi): \mathbb{R}^{3} \backslash \mathscr{A} \longrightarrow \mathbb{H}_{\mathbb{C}}^{2}
$$

which differs from $I$ by the boundary term

$$
B_{\Omega}(U)=\int_{\partial \Omega} \frac{\partial \ln \rho}{\partial N}(2 U-\ln \rho) d S,
$$

where $N$ is the outward pointing unit normal to $\partial \Omega$. Consequently for both $I$ and $H$ the associated variational equations are the harmonic map equations, with target space the two-dimensional complex hyperbolic space. Hence the target manifold satisfies the convexity conditions of [16] (see Remark (i), p. 5 there). For compact $\Omega$ away from the axis we can thus conclude from [16] that action minimisers of $H_{\Omega}$ with Dirichlet boundary conditions exist, are 
smooth, and satisfy the variational equations. It is also well known (see [5] and references therein) that solutions of the Dirichlet boundary value problem are unique when the target manifold has negative sectional curvature, which is the case here. All this implies that $(\tilde{U}, \tilde{v}, \tilde{\chi}, \tilde{\psi})$, with its own boundary data, minimizes the action integral $H$, and consequently of $I$, over the sets

$$
\mathscr{C}_{\sigma, \epsilon}:=\{\rho \geq \epsilon\} \cap\{\sigma / 2 \leq r \leq 2 / \sigma\} .
$$

In particular, since the maps $\left(\theta_{\sigma, \epsilon}\right)$ and $(\tilde{\theta})$ coincide on $\partial \mathscr{C}_{\sigma, \epsilon}$ but are manifestly distinct, we conclude that

$$
I_{\mathscr{C}_{\sigma, \epsilon}}\left(U_{\sigma, \epsilon}, v_{\eta, \epsilon}, \chi_{\eta, \epsilon}, \psi_{\eta, \epsilon}\right)>I_{\mathscr{C}_{\sigma, \epsilon}}(\tilde{U}, \tilde{v}, \tilde{\chi}, \tilde{\psi})
$$

In fact the maps under consideration coincide on the closure of the complement of $\mathscr{C}_{\sigma, \epsilon}$ and therefore

$$
I^{\sigma, \epsilon}>\tilde{I}
$$

Recalling (2.28) we obtain

$$
I^{\sigma}=\lim _{\epsilon \rightarrow 0} I^{\sigma, \epsilon} \geq \lim _{\epsilon \rightarrow 0} \tilde{I}=\tilde{I} .
$$

Returning to the proof of Theorem 1.1, Lemmata 2.4 and 2.5 yield

$$
I=\lim _{\sigma \rightarrow 0} I^{\sigma} \geq \tilde{I}
$$

and the result is a consequence of Remark 2.1 followed by a duality rotation.

\section{Concluding remarks}

The study of Dain inequalities is still in an early stage with important questions still needing to be settled even for pure vacuum; also, some impressive generalization can be easily formulated and justified by the heuristic argument presented in the introduction. We finish this chapter by addressing some of this issues: 
1. Extreme Kerr-Newman as a minimum of the mass functional. Our class of data does not include extreme Kerr-Newman and consequently eliminates a priori the possibility of establishing it as the unique minimum for the mass functional. This difficulty is not present in Dain's original work where a class of "Brill" data is considered; however, this is done at the cost of a considerably longer list of (stronger) technical assumptions, some of which are derivable properties of asymptotic flatness and the existence of multiple ends as was first observed and established in [3]. To obtain the desired result within the spirit of the program initiated by Chruściel one could start by generalizing the results in [3] for data allowing for both asymptotically flat and asymptotically cylindrical ends, and then try to adapt the arguments presented here.

From what as been said, we expected inequality (1.3) to be strict within the class of data considered in this work.

2. Multiple asymptotically flat ends. Even for vacuum the question of multiple ends requires further work. Although a Dain inequality was already established in [5] it depends on a function of the angular momenta for which an explicit expression remains unknown for all $N>2$, where $N$ is the number of asymptotically flat ends. This is clearly an unsatisfactory situation since the Penrose-like argument, presented at the beginning of this paper, provides evidence that the unknown function should simply be the square root of the total angular momentum. In fact, for the two body problem, $N=3$, such expectation as been recently supported by numerical evidences [14].

One also expects the ideas in [5] to generalize to electro-vacuum by using the methods developed here, but in this case it seems hard to speculate what the exact expression for the lower bound function, this time of both angular momenta and Maxwell charges, should be. This problem is related to the fact that the Majumdar-Papapetrou metrics provide the existence of regular and extreme multiple black hole solutions; analogous difficulties have been found for the Penrose inequality [20].

3. Asymptotically electro-vacuum data. The heuristic argument leading to the Dain inequality presented here works for other data, involving far more general matter models: axisymmetric asymptotically 
electro-vacuum initial data whose domain of outer communications becomes electro-vacuum asymptotically with time. Establishing a Dain inequality in such generality would be quite impressive but, at this moment, such a goal seems unreachable.

Acknowledgements: The author is grateful to Piotr Chruściel for making him a part of this challenging project. He would also like to thank José Natário, Paul Tod and Harvey Reall for numerous comments on a previous version of this work.

\section{A Decay rates for Extreme Kerr-Newman}

\begin{tabular}{cc}
\hline \hline$r \rightarrow 0$ & $r \rightarrow+\infty$ \\
\hline$\tilde{U}=\log (r)+O(1)$ & $\tilde{U}=-\frac{m}{r}+O\left(r^{-2}\right)$ \\
$\tilde{\chi}=\rho^{2} O\left(r^{-2}\right)=O(1)$ & $\tilde{\chi}=\rho^{2} O\left(r^{-3}\right)=O\left(r^{-1}\right)$ \\
$\tilde{\psi}=O(1)$ & $\tilde{\psi}=\rho O\left(r^{-2}\right)=O\left(r^{-1}\right)$ \\
$\partial_{\rho} \tilde{\chi}=\rho O\left(r^{-2}\right)$ & $\partial_{\rho} \tilde{\chi}=\rho O\left(r^{-3}\right)$ \\
$\partial_{\rho} \tilde{\psi}=\rho O\left(r^{-2}\right)$ & $\partial_{\rho} \tilde{\psi}=\rho O\left(r^{-2}\right)$ \\
$|D \tilde{\chi}|_{\delta}=\rho O\left(r^{-2}\right)$ & $|D \tilde{\chi}|_{\delta}=\rho O\left(r^{-3}\right)$ \\
$|D \tilde{\psi}|_{\delta}=O\left(r^{-1}\right)$ & $|D \tilde{\psi}|_{\delta}=O\left(r^{-1}\right)$ \\
$\tilde{v}=O(1)$ & $\tilde{v}=O(1)$ \\
$\partial_{\rho} \tilde{v}=\rho O\left(r^{-2}\right)$ & $\partial_{\rho} \tilde{v}=\rho O\left(r^{-2}\right)$
\end{tabular}

Table 1: Decay rates for Extreme Kerr-Newman

\section{B Identity (2.16)}

Here we present the computations leading to equation (2.16). Let $\theta=v, \chi, \psi$ and write

$$
\theta_{\sigma}:=f_{\sigma} \theta+\left(1-f_{\sigma}\right) \tilde{\theta}=f_{\sigma}(\theta-\tilde{\theta})+\tilde{\theta} \text {. }
$$


Then

$$
\begin{aligned}
\lambda_{\sigma}:= & D v_{\sigma}+\chi_{\sigma} D \psi_{\sigma}-\psi_{\sigma} D \chi_{\sigma} \\
= & D f_{\sigma}(v-\tilde{v})+f_{\sigma} D(v-\tilde{v})+D \tilde{v} \\
& +\left[f_{\sigma}(\chi-\tilde{\chi})+\tilde{\chi}\right]\left[D f_{\sigma}(\psi-\tilde{\psi})+f_{\sigma} D(\psi-\tilde{\psi})+D \tilde{\psi}\right] \\
& -\left[f_{\sigma}(\psi-\tilde{\psi})+\tilde{\psi}\right]\left[D f_{\sigma}(\chi-\tilde{\chi})+f_{\sigma} D(\chi-\tilde{\chi})+D \tilde{\chi}\right] \\
= & D \tilde{v}+\tilde{\chi} D \tilde{\psi}-\tilde{\psi} D \tilde{\chi}+D f_{\sigma}(v-\tilde{v})+f_{\sigma} D(v-\tilde{v}) \\
& +f_{\sigma} D f_{\sigma}(\chi-\tilde{\chi})(\psi-\tilde{\psi})-f_{\sigma} D f_{\sigma}(\psi-\tilde{\psi})(\chi-\tilde{\chi}) \\
& +f_{\sigma}^{2}(\chi-\tilde{\chi}) D(\psi-\tilde{\psi})-f_{\sigma}^{2}(\psi-\tilde{\psi}) D(\chi-\tilde{\chi}) \\
& +f_{\sigma}(\chi-\tilde{\chi}) D \tilde{\psi}-f_{\sigma}(\psi-\tilde{\psi}) D \tilde{\chi} \\
& +D f_{\sigma} \tilde{\chi}(\psi-\tilde{\psi})-D f_{\sigma} \tilde{\psi}(\chi-\tilde{\chi}) \\
& +f_{\sigma} \tilde{\chi} D(\psi-\tilde{\psi})-f_{\sigma} \tilde{\psi} D(\chi-\tilde{\chi}) \\
= & \tilde{\lambda}+D f_{\sigma}(v-\tilde{v})+D f_{\sigma}(\tilde{\chi} \psi-\tilde{\psi} \chi) \\
& +f_{\sigma}^{2}\{(\chi-\tilde{\chi}) D(\psi-\tilde{\psi})-(\psi-\tilde{\psi}) D(\chi-\tilde{\chi})\} \\
& +f_{\sigma}\{(\chi-\tilde{\chi}) D \tilde{\psi}-(\psi-\tilde{\psi}) D \tilde{\chi}\} \\
& +f_{\sigma} \underbrace{\{D(v-\tilde{v})+\tilde{\chi} D(\psi-\tilde{\psi})-\tilde{\psi} D(\chi-\tilde{\chi})\}}_{*} .
\end{aligned}
$$

The last line reads

$$
\begin{aligned}
* & =-D \tilde{v}-\tilde{\chi} D \tilde{\psi}+\tilde{\psi} D \tilde{\chi}+D v+\tilde{\chi} D \psi-\tilde{\psi} D \chi \\
& =\lambda-\tilde{\lambda}-(\chi-\tilde{\chi}) D \psi+(\psi-\tilde{\psi}) D \chi
\end{aligned}
$$

and the desired identity follows.

\section{References}

[1] D. Brill, On the positive definite mass of the Bondi-Weber-Wheeler timesymmetric gravitational waves, Ann. Phys. 7 (1959), 466-483.

[2] Y. Choquet-Bruhat, General relativity and the Einstein equations, Oxford Mathematical Monographs. Oxford University Press, Oxford (2009). 
[3] P.T. Chruściel, Mass and angular-momentum inequalities for axisymmetric initial data sets. I. Positivity of mass, Annals Phys. 323 (2008), 2566-2590, arXiv:gr-qc/0710.3680.

[4] _ On completeness of orbits of Killing vector fields, Classical Quantum Gravity 10 (1993), no. 10, 2091-2101, arXiv:gr-qc/9304029.

[5] P.T. Chruściel, Y. Li, and G. Weinstein, Mass and angular-momentum inequalities for axi-symmetric initial data sets. II. Angular momentum, Annals Phys. 323 (2008), 2591-2613, arXiv:gr-qc/0712.4064v2.

[6] P. Chruściel and J.L. Costa, On Uniqueness of Stationary Vacuum Black Holes, Gómétrie Différentielle, Physique Mathématique, Mathématique et Société, Volume en l'honneur de Jean Pierre Bourguignon (O. Hjazi, éditeur), Astérisque 321, 2008, p. 195-265. arXiv:gr-qc/0806.0016.

[7] _ Mass, angular-momentum, and charge inequalities for axisymmetric initial data, Class. Quantum Grav. 26 (2009) 235013, arXiv:grqc/0909.5625.

[8] J. Lopes Costa, On the classification of stationary electro-vacuum black holes, Class. Quantum Grav. 27 (2010) 035010, arXiv:grqc/0912.0834v1.

[9] _ A Dain inequality with charge, arXiv:gr-qc/0912.0838.

[10]__ On black hole uniqueness theorems, DPhil Thesis, Oxford, 2010.

[11] S. Dain, A variational principle for stationary, axisymmetric solutions of Einstein's equations, Classical Quantum Gravity 23 (2006), no. 23, 6857-6871. arXiv:gr-qc/0508061.

[12] _ Proof of the angular momentum-mass inequality for axisymmetric black holes, Jour. Diff. Geom. 79 (2006), 33-67, arXiv:gr-qc/0606105.

[13] _ , The inequality between mass and angular momentum for axially symmetric black holes, Int. Jour. Modern Phys. D 17 (2008), 519-523, arXiv:gr-qc/0707.3118.

[14] _ Numerical evidences for the angular momentum-mass inequality for multiple axially symmetric black holes, arXiv:gr-qc/0905.0708. 
[15] G.W. Gibbons and G. Holzegel, The positive mass and isoperimetric inequalities for axisymmetric black holes in four and five dimensions, Class. Quantum Grav. 23 (2006), 6459-6478, arXiv:gr-qc/0606116.

[16] S. Hildebrandt, H. Kaul and K. Widman, An existence theorem for harmonic mappings of Riemannian manifolds, Acta Math. 138 (1977), no. $1-2,1-16$.

[17] R. Penrose, Gravitational collapse: the role of general relativity, Reprinted from Rivista del Nuovo Cimento 1969, Numero Speziale I, 252-276. Gen. Relativity Gravitation 34 (2002), no. 7, 1141-1165.

[18] R. Wald, Gravitational collapse and cosmic censorship, Black holes, gravitational radiation and the universe, 69-85, Fund. Theories Phys., 100, Kluwer Acad. Publ., Dordrecht, (1999), arXiv:gr-qc/9710068.

[19] G. Weinstein, N-black hole stationary and axially symmetric solutions of the Einstein/Maxwell equations, Commun. Part. Diff. Eqs. 21 (1996), 1389-1430.

[20] G. Weinstein and S. Yamada, On a Penrose inequality with charge, arXiv:math.dg/0405602. 